\title{
Variation of drag coefficient with wind wave development
}

\author{
ZHAN G Zifan（张子范） and L I Jiachun (李家春) \\ (Institute of Mechanics, Chinese Academy of Sci nces, Beijing 100085, China)
}

Received Octobe: 26,199 ?

\begin{abstract}
Turbulent air flows over teveloping wind waves is: tne air-sea boundary layer are numerically simulated without considering wave breaking. Influences of wind waves on air flows are considered using a model of significant wave and surface roughness, with a formula proposed for calculating the surface roughness. $k-\epsilon$ model is adopted to simulate turbulent flows. The results of the drag coefficient and turbulence characteristics agree well with the observations.
\end{abstract}

Key words : drag coefficient, wind uaves, $\mathrm{k}-\epsilon$ turbulence model, air-sea interaction.

Momentum, energy and mass exchange between air and sea is a fundamental problem in many fields of research, such as climate change, wave forecast and ocean circulation. In order to determine the momentum flux through interface we should study small scale processes in the airsea boundary layer. It is a problem of air-sea interaction including complex waves resolution and turbulence, so the quantitative description is still not satisfactory up to now. The momentum flux $\mathrm{T}$ can be calculated by a bulk formula : $\mathrm{T}=\rho C_{\mathrm{D}} U_{10}^{2}$, where $\rho$ is the air density, $U_{10}$ is the windspeed at $10 \mathrm{~m}$ above sea surface, and $C_{\mathrm{D}}$ is named the drag coefficient on the sea surface.

There have been many marine observations and theoretical analyses to study the drag coefficient, but the results are sporadic substantially. The reason is that the momentum flux is related with not only windspeed, but also other oceanic conditions, such as atmosphere stability, wave developing degree and wave breaking. So the drag coefficient $C_{\mathrm{D}}$ also varies with windspeed and wave development when the atmosphere is neutrally stratified. As wind blows over sea surface, momentum is transported to sea water and then waves are generated. Reversely, waves influence air flows above it. Waves in different developing period have different structures, which make the momentum flux on air sea interface change. Developing period of wind waves determined by fetch and duration is represented by wave age $C_{\mathrm{p}} / U *$ or $C_{P} / U_{10}$, where $C_{P}$ is the phase velocity of wave spectrum peak; $U *$ is the friction velocity. Analyzing the measured data on Lake Ontario, Donelan ${ }^{[1]}$ concluded that the drag coefficient decreases with the developing of wind waves.

Measurement shows that vertical profile of windspeed is of logarithmic form. Then $C_{\mathrm{D}}$ can be calculated by the following equation: $C_{\mathrm{D}}=\left[\mathrm{K} / \ln \left(z^{\prime} / z_{0}\right)\right]^{2}$, where $\mathrm{K}$ is the Karman constant, and $z_{0}$ is the surface roughness of ocean. Charnock ${ }^{[2]}$ proposed $z_{0}=\alpha \frac{U^{2}{ }_{*}}{g}$, where $g$ is the gravity acceleration, $\mathbf{a}$ is the Charnock constant determined by measurement. Maat et al. ${ }^{[3]}$ proposed

* Project supported by the National Natural Science Foundation of China (Grant No. 19332010) . 
that $\boldsymbol{\alpha}$ is related to wave age by suggesting a formula: $z_{0}{ }^{*}=\frac{g z_{0}}{U^{2}{ }_{*}}=\mu\left(C_{\mathrm{p}} / U_{*}\right)^{n}$, where $\mu=0$. $8 ; n=-1$. Sea surface roughness $z_{0}$ represents the influence of surface waves on air flow. It is a function of wave age, meaning that the developing of wind waves changes the air-sea momentum exchange. From $z_{0}$ we can obtain the formula $C_{\mathrm{D}}: \frac{U_{10}^{2}}{10 g}\left(C_{\mathrm{p}} / U_{*}\right)^{n}=\mu^{-1} C_{\mathrm{D}}^{\frac{n}{2}-1} \exp \left(-\mathrm{K} / \sqrt{C_{\mathrm{D}}}\right)$. It means that $C_{\mathrm{D}}$ is related not only to $U_{10}$, but also to the wave age $C_{\mathrm{P}} / U *$.

Random and irregular wind waves usually are described by wave spectrum. Research on the roles of different frequency parts of spectrum is important for understanding the mechanism of airsea interaction. Kitaigorodskii ${ }^{[4]}$ found that the sea surface roughness is determined by high frequency gravity and capillary waves. They play a dominant role in momentum transfer from wind to waves. Donelan ${ }^{[1]}$ considered both the shape drag induced by low frequency waves and the friction drag induced by high frequency waves. Geernaert ${ }^{[5-7]}$ compared these roughness models and concluded that Kitaigorodskii' s model simulates variation of $C_{\mathrm{D} N}$ more accurately. This means that the high frequency part of wind waves is dominant in momentum exchange. The result of Makin et al. ${ }^{[8]}$ agrees well with this conclusion. However, the theory about the role of different frequency parts of wind waves in air-sea interaction is still not completed so far because of many difficulties, such as wind wave coupled interaction, wave-wave interaction and turbulence.

Since observations over sea are very difficult, the situation hinders further research. With the development of CFD, numerical simulation is a usef ul tool for the research on air sea interaction. Chalikov $^{[9,10]}$, Le Ngocly ${ }^{[11]}$, Maat and Makin ${ }^{[12]}$ have done some researches, which are helpf ul to understanding the moment um transfer and turbulence field near the air-sea interface. However, these numerical models are different from the real field of air-sea boundary layer: the influence of waves on air flow is not considered; turbulence model is too simple; and there are few works of numerical study on air-sea momentum exchange. Zhang and $\mathrm{Li}^{[13]}$ studied the influence of developed wind waves on interface momentum exchange by applying the $k-\epsilon$ turbulence model. Variation of the drag coefficient with windspeed was obtained, which is in good agreement with the observational data. In order to study the influence of wave development on momentum exchange and turbulence field above waves, this paper numerically simulates the turbulence air flow over developing wind waves, and $k-\epsilon$ model is also adopted. Influence of wind waves on air flows is considered by a model of significant wave and surface roughness. Variation of the drag coefficient and turbulence flow due to windspeed and wave age is compared with the observations.

\section{Mathematical model and numerical simulation}

\section{1 Sea surface roughness and wind wave developing}

Moderate and low frequency waves have larger wave height, wave length, wave speed and smaller slope. Air turbulence structure is correlated with wave phase and shape; that is to say, moderate and low frequency waves change the large-scale turbulent movement and also influence the air-sea interaction. High frequency waves have smaller wave height, wave length, wave speed and larger slope and they change the small-scale structure of turbulence and then influence the momentum exchange. Considering the influence of all kinds of waves, significant wave and wave surface roughness are used in numerical simulation. The effective wave represents the influence of large-scale wind waves, the wave surface roughness $z_{\mathrm{s}}$ represents the influence of high frequency 
or small-scale part of wind waves. $\omega=2 \omega_{p}$ is taken as the critical frequency to demarcate the wave spectrum. $z_{\mathrm{s}}$ and $z_{0}$ are introduced by different meanings. $z_{0}$ is the effective roughness of sea surface and represents the influence of sea wave condition on air flow.

The JONSWAP spectrum describes the developing wind waves due to wind speed and fetch:

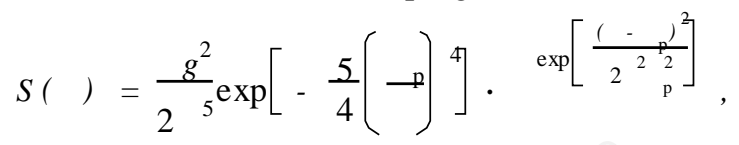

where $\mathbf{\alpha}=0.076 \tilde{x}^{0.22} ;$ the nondimensional fetch $\tilde{x}=\frac{g x}{U_{10}^{2}}, ; x$ is the wind fetch; $U_{10}$ is the $10 \mathrm{~m}$ high windspeed; the nondimensional peak frequency of spectrum $\tilde{\omega}_{\mathrm{p}}=\frac{U_{10} \omega_{\mathrm{p}}}{g}=22 \tilde{x}^{-0.33}, \mathrm{~V}$ $=3.3$; when $\omega>\omega_{\mathrm{p}} \sigma=0.09$; otherwise $\sigma=0.07$. According to the JONSWAP spectrum, the significant wave height, length and speed can be calculated: $H_{1 / 3}=4 \sqrt{\int_{0}^{\infty} S(\omega) \mathrm{d} \omega, \Lambda_{1 / 3}}=$ $0.00524 U_{10}^{0.68} x^{0.66}, C_{1 / 3}=0.0905 U_{10}^{0.34} x^{0.33}$.

The JONSWAP spectrum was firstly obtained by observing the North Sea deep water waves and the largest fetch is about $160 \mathrm{~km}$. Because of the limited range, the spectrum is not accurate enough to describe the large fetch wind waves and developed waves. So the P-M spectrum is adopted to describe the developed waves:

$$
S(\omega)=0.5 a g^{2} \omega^{-5} \exp \left[-0.74\left(\frac{g}{\omega \omega}\right)^{4}\right] \text {, }
$$

where $\mathrm{a}=8.1 \times 10^{-3} ; g$ is the gravity acceleration; $U$ is the $19.5 \mathrm{~m}$ high windspeed ; the peak frequency of $\mathrm{P}-\mathrm{M}$ spectrum is $\omega_{\mathrm{p}}=0.877 \mathrm{gU}^{-1}$; the significant wave height is $H_{1 / 3}=0.021$ $4 U^{2}$; significant wave period is $T_{1 / 3}=0.937 T_{\mathrm{p}}=0.684 U$. The wave slope is low, so the significant wave can be regarded as the small wave. Far from the sea surface the windspeed is assumed as the logarithmic profile. Then the significant wave height, length and speed can be calculated: $H_{1 / 3}=0.0251 U_{10}^{2}, \lambda_{1 / 3}=0.856 U_{10}^{2}, C_{1 / 3}=1.156 U_{10}$.

The mean-square- root wave amplitude of small-scale high frequency wind waves can be calculated $: \sigma_{\eta}=\left[2 \int_{2 \omega_{p}}^{\infty} S(\omega) \mathrm{d} \omega\right]^{1 / 2}$. Differing from the land surface roughness, the roughness over moving sea surface is determined by not only the shapes of roughness elements, but also their movement. The wave surface roughness $z_{\mathrm{s}}$ represents the contribution of high frequency waves to momentum transfer. It is related to $\sigma_{\eta}$ and wave age $C_{\mathrm{p}} / U_{*}$. After dimensional analysis the formula is obtained: $F\left(\frac{z_{\mathrm{s}}}{\sigma_{\eta}}, \frac{C_{\mathrm{p}}}{U_{10}}\right)=0$, or $z_{\mathrm{s}}=f\left(\frac{C_{\mathrm{p}}}{U_{10}}\right) \sigma_{\eta}$. According to the observation and numerical simulation, it is concluded that

$$
z_{\mathrm{s}}=A \mathrm{e}^{-B C_{\mathrm{p}} / U_{10} \sigma_{\eta}},
$$

where $A=16.0$, and $B=6.5$. As for developed waves, $z_{\mathrm{s}}$ is only the function of $\sigma_{\eta}$ :

$$
z_{\mathrm{s}}=\beta \sigma_{\eta},
$$

where $\beta=7.07 \times 10^{-3}$ corresponding to the wave age $C_{\mathrm{p}} / U_{10}=1.19$ in formula (3) .

\subsection{Turbulence model}

Generally speaking, air-sea boundary layer flows are turbulent. Therefore they can be de- 
scribed by the Reynolds averaged equations and the two-equation $k-\epsilon$ model is used to approximate them. Although the lower boundary is curvilinear, $k-\epsilon$ model can also be applied well because of the small curvature in this case. The numerical model of flow field is two dimensional, $x_{1}$ is the horizontal coordinate, which is parallel with wind, $x_{3}$ is the vertical coordinate, and $x_{3}$ $=0$ is the mean height of surface wave.

1.2.1 Governing equations. The Reynolds equation system looks like

$$
\frac{\partial u_{i}}{\partial t}+u_{j} \frac{\partial u_{i}}{\partial x_{j}}=-\frac{1}{\rho} \frac{\partial p}{\partial x_{i}}+\mathrm{v} \frac{\partial^{2} u_{i}}{\partial x_{j} \partial x_{j}}-\frac{\partial \overline{u_{i}^{\prime} u_{j}^{\prime}}}{\partial x_{j}}
$$

where $u_{i}$ is the mean velocity; $u_{i}^{\prime}$ is the perturbation velocity, $i, j=1,3 ; p=P_{0}+\rho g x_{3}$.

The continuous equation can be written as

$$
\frac{\partial u_{i}}{\partial x_{i}}=0
$$

And $k €$ model equations are

$$
\begin{aligned}
& \text { - } \overline{u_{i}^{\prime} u_{j}^{\prime}}=v_{t}\left(\frac{\partial u_{i}}{\partial x_{j}}+\frac{\partial u_{i}}{\partial x_{i}}\right)-\frac{2}{3} \delta_{i j}, \\
& \mathrm{v}_{t}=G_{\mu} \frac{k^{2}}{\epsilon} \text {, } \\
& \frac{\partial k}{\partial t}+u_{j} \frac{\partial k}{\partial x_{j}}=\frac{\partial}{\partial x_{j}}\left[\left(v+v_{t} / \sigma_{k}\right) \frac{\partial k}{\partial x_{j}}\right]+G_{k}-\epsilon, \\
& \frac{\mathfrak{\varpi}}{\partial t}+u_{j} \frac{\mathfrak{\varpi}}{\partial x_{j}}=\frac{\partial}{\partial x_{j}}\left[\left(\mathbf{v}+\mathrm{v}_{t} / \sigma_{\epsilon}\right) \frac{\mathfrak{\varpi}}{\partial x_{j}}\right]+C_{1} \in G_{k} / k-C_{2} \epsilon^{2} / k \text {, } \\
& G_{k}=\mathbf{v}_{t}\left(\frac{\partial u_{i}}{\partial x_{j}}+\frac{\partial u_{i}}{\partial x_{i}}\right) \frac{\partial u_{i}}{\partial x_{j}},
\end{aligned}
$$

where $G_{\mu}=0.09, \sigma_{k}=1.0, \sigma_{\epsilon}=1.0, C_{1}=1.44$, and $C_{2}=1.92$.

1.2.2 Boundary conditions. The coordinate system is fixed on the wave surface, so the flow field becomes steady. The lower boundary is the regular cosine wave, so the calculated domain is regarded as a period of field; thus the periodical boundary conditions can be imposed on two lateral sides. Near the sea surface the influence of waves on flow field is apparent and decays rapidly with the increasing height. So it can be neglected at the top of domain, which is at the height of half wave length (the horizontal windspeed $u$ is assumed to be constant) ; the pressure is also a constant $p_{0}$; the vertical windspeed $w$, turbulence kinetic energy $k$ and dissipation $\epsilon$ are invariant with height, or their vertical derivatives vanish.

The sea surface velocity includes wave movement and water drift. The drift velocity is small and can be neglected. The turbulence energy and velocity vary rapidly near the wall, so in numerical simulation grids should be finer. Near wall turbulence is weak and the molecular viscosity is important, so the assumption of high Reynold number is not proper there. In order to simulate the turbulence near the wall, a wall function assumption is adopted: near the wall only the velocity parallel with the wall is in effect ; turbulence energy generation and dissipation are in balance ; the Reynolds stress equals wall stress and is homogeneous. This wall function assumption is obtained after studying turbulence field near the wall, and is available for turbulence near the rough wall. Because the significant wave slope is very small, this assumption can also apply to turbulence near the air-sea interface. 
The coordinate transformation is as follows: $x=x_{1}-C_{1 / 3} t, z=x_{3} ; u=u_{1}-C_{1 / 3}, w$ $=u_{3}$. Therefore the boundary conditions can be written.

(i) At the top of the field, the air pressure, velocity, turbulence kinetic energy and dissipation at the height of half wave length are

$$
\begin{aligned}
\left.p\right|_{\lambda / 2} & =P_{0}, \\
\left.u\right|_{\lambda / 2} & =U_{\lambda / 2}-C_{1 / 3}, \\
\left.\frac{\partial w}{\partial z}\right|_{\lambda / 2} & =\left.\frac{\partial k}{\partial z}\right|_{\lambda / 2}=\left.\frac{\partial \notin}{\partial z}\right|_{\lambda / 2}=0 .
\end{aligned}
$$

(ii) At the bottom of the field, the velocity on wave surface is

$$
\begin{aligned}
& \left.u\right|_{0}=\frac{H_{1 / 3}}{2} \sqrt{\frac{\pi}{2}} \cos \left(\frac{\pi x}{\lambda_{1 / 3}}\right)-C_{1 / 3}, \\
& \left.w\right|_{0}=-\frac{H_{1 / 3}}{2} \sqrt{\frac{\pi}{g}} \sin \left(\frac{\pi x}{\lambda_{1 / 3}}\right) .
\end{aligned}
$$

A non-orthogonal body-fitted-coordinate system is adopted. Then the velocity near interface parallels it. The boundary conditions of velocity are

$$
\begin{aligned}
& \left.u\right|_{w}=\sqrt{\frac{\sqrt{g H_{1 / 3}^{2}}+C_{1 / 3}^{2}-C_{1 / 3} H_{1 / 3}}{\sqrt{2 \lambda_{1 / 3}}} \cos \left(\frac{2 \pi x}{\lambda_{1 / 3}}\right)}, \\
& \left.w\right|_{w}=0 .
\end{aligned}
$$

According to the wall function assumption we have, in the first grid point near the interface $\left(z=z_{1}\right)$,

$$
\begin{aligned}
u-u_{w} & =u *\left[\frac{1}{\mathrm{~K}} \ln z^{+}+5.24-\Delta u^{+}\left(r^{+}\right)\right], \\
k & =\frac{\mathrm{T}_{w}}{C_{\mu}^{1 / 2} \rho}=\frac{u^{2} *}{C_{\mu}^{1 / 2}}, \\
\epsilon & =\frac{k^{3 / 2} C_{\mu}^{3 / 4}}{\mathrm{~K} z_{1}}=\frac{u^{3} *}{\mathrm{~K} z_{1}},
\end{aligned}
$$

where $u *$ is the friction velocity; $u_{w}$ is the velocity at the interface, $u_{w}=u \mid{ }_{w} ; z^{+}=z u * / \mathrm{V}$; $\Delta u^{+}=2.83 \ln r^{+}-4.56, r^{+}=z_{s} u * / \mathrm{V}$, when $r^{+}<5 \Delta u^{+}=0 ; \mathrm{K}=0.4$ is the Karman constant ; $\mathbf{T}_{w}$ is the wall shear stress; $z_{1}$ is determined from $z_{1}^{+}=11.0 ; u *$ is unknown, so iteration is needed in calculation. Assuming that the velocity of the first grid point $u$ is known, $u *$ can be calculated from (19), and so $k, \in$ can be obtained from (20) and (21). Then $u$ can be calculated again after we solve the control equations. Iterate it for several times until $\left(u_{*}^{n+1}-u_{*}^{n}\right) / u^{n}{ }_{*}<$ $10^{-3}$. The distance of the first grid point from interface is obtained from $z_{1}^{+}=z_{1} u * / V=11.0$. So after each iteration, new values of $u *$ and $z_{1}$ are obtained until convergence.

(iii) Boundary conditions at two lateral sides are

$$
\begin{aligned}
p_{l} & =p_{r}, \quad u_{l}=u_{r}, \\
w_{l}=w_{r}, \quad k_{l} & =k_{r}, \quad \epsilon_{l}=\epsilon_{r} .
\end{aligned}
$$

\subsection{Computational scheme}

The finite volume method is preferred in the numerical calculation. The calculated domain is divided into finite number of control volumes by grids. According to the conservation laws of 
mass, momentum, energy or other scalar quantities in each control volume, the general equation system of the fluid dynamics is obtained:

$$
\frac{\partial(\rho \phi)}{\partial t}+\operatorname{div}(\rho u \phi)=\operatorname{div}(\Gamma \operatorname{grad} \phi)+S_{\phi} .
$$

It can be discretized into an algebraic equation system. For two-dimensional field, it is

$$
a_{p} \phi_{p}=a_{e} \phi_{e}+a_{w} \phi_{w}+a_{n} \phi_{n}+a_{s} \phi_{s}+b \text {. }
$$

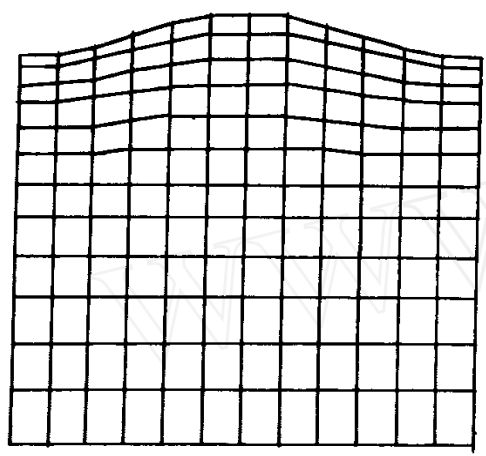

Fig. 1. Calculating grids

The non-orthogonal body-fitted coordinate grids are adopted (fig. 1). Because of non-orthogonal nature, the equations should be transformed into an appropriate form. All the derivation is omitted here for simplicity. For more details, see reference [13].

1. 4 Calculation of the drag coefficient $C_{\mathrm{D}}$

The momentum flux can be calculated in the following way:

$$
\mathbf{T}=\mathbf{T}_{w} \cos \theta+p \sin \theta,
$$

where $\mathbf{T}_{w}$ is the friction stress at the interface; $p$ is the pressure; $\theta$ is the angle between the wave surface and horizontal orientation. According to the wall function assumption $\mathbf{T}_{w}$ can be calculated $: \mathrm{T}_{w}=\mid \mathrm{P} C_{\mu}^{1 / 2}$, where $k$ is the turbulence kinetic energy in the first grid point from interface. The momentum flux at sea surface can be obtained by intergrating them over a period of wave and making average. Then $C_{\mathrm{D}}$ can be calculated by $C_{\mathrm{D}}=\frac{\mathrm{T}}{\rho U_{10}^{2}}$.

\section{Results and discussion}

The air turbulence field over wind sea has been calculated under different windspeeds. $10 \mathrm{~m}$ high windspeeds are $5,7.5,10,12.5$ and $15 \mathrm{~m} / \mathrm{s}$. Under 5 and $7.5 \mathrm{~m} / \mathrm{s}$ wind, calculated fetches are $1,2,5,30 \mathrm{~km}$, and $100 \mathrm{~km}$ fetch is also calculated for $7.5 \mathrm{~m} / \mathrm{s}$ wind. When windspeeds are $10,12.5,15 \mathrm{~m} / \mathrm{s}$, and the fetches are $2,5,30,100$ and $200 \mathrm{~km}$. In addition, the air flows above the developed waves are also calculated.

The wave surface roughness $z_{\mathrm{s}}$ and sea surface effective roughness $z_{0}$ are shown in fig. 2 , in which curves $1-3$ represent the results under 5,10 and $15 \mathrm{~m} / \mathrm{s}$ windspeed respectively. Fig. 2 shows that $z_{\mathrm{s}}$ is about 10 times higher than $z_{0}$. It can be explained by the different meanings of them. High frequency waves are regarded as roughness elements moving on the significant wave surface, so $z_{\mathrm{s}}$ represents the contribution of the high frequency part of wind waves to air-sea interaction. $z_{0}$ is the effective roughness of sea surface under the assumption of windspeed logarithmic distribution, so it represents the influence of sea surface status, including the whole part of wave spectrum on air turbulence flows. With the increase of the wave age, $z_{0}$ decreases. This reflects the influence of sea waves on air flow and interface course. When the wind waves are dever oping, their structures are changing, thus affecting the interface course, and the moment um flux decreases. The high frequency part of wind waves plays a significant role, so $z_{\mathrm{s}}$ also decreases with the wave age increasing. Approximately $z_{\mathrm{s}}$ and $z_{0}$ decrease linearly with the wave age.

The change of air flow structure with wind wave developing is shown in fig. 3 . The profiles 

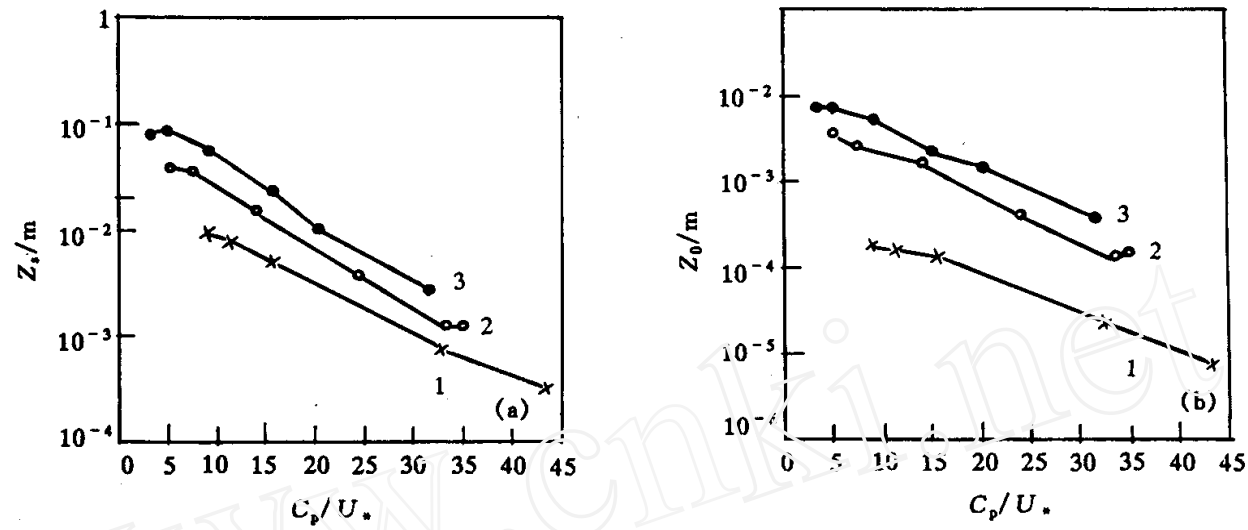

Fig. 2. Variztion of wave surface roughness $z_{\mathrm{s}}$ and sea surface effective roughness $z_{0}$ with wind wave developing.

are the vertical distribution above the wave crest when the windspeed is $10 \mathrm{~m} / \mathrm{s}$. Curves $1,2,3$ and 4 represent the different fetches of $2,5,30,100 \mathrm{~km}$. Fig. 3 (a) is the vertical profiles of
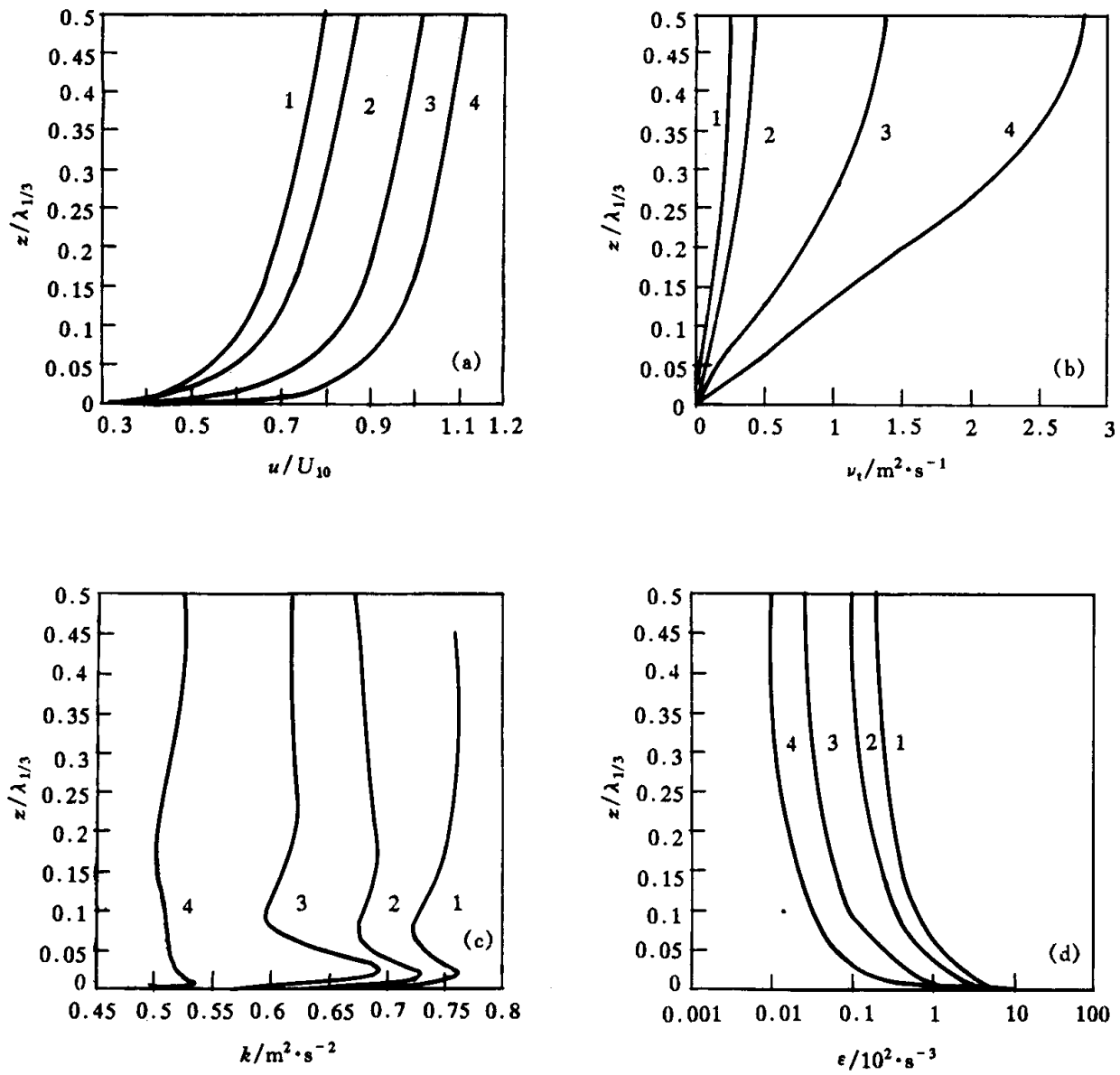

Fig. 3. Variation of air turbulence structure above waves with wind-wave developing. 
windspeed. The profiles are of the logarithmic form at the large height, or far from the sea surface, whereas the profiles deviate from the logarithmic form as they approach the sea surface. This shows the influence of waves on large scale of air turbulence. Figs. 3(b) -3 (d) are the profiles of turbulence eddy viscosity coefficient, turbulence kinetic energy and dissipation. With the wind wave developing, the turbulence eddy viscosity increases and is much higher than the molecular viscosity coefficient. This means that the turbulence exchange in air-sea boundary layer plays a dominant role. The decrease in turbulence energy and dissipation shows that the turbulence intensity becomes lower with the wave development. Because of the dominant role of turbulence, the reduction in turbulence intensity means the decreasing air-sea momentum exchange.

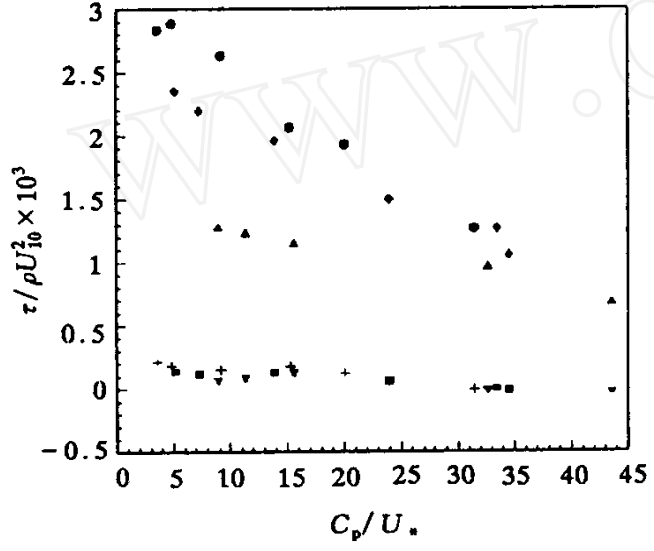

Fig. 4. Turbulence friction drag and wave shape drag over sea surface.

Figure 4 shows the variation turbulence friction drag and wave shape drag with the wind speed and wave age. The dots, diamonds and triangles represent the turbulence friction drag under the windspeeds 5,10 and $15 \mathrm{~m} / \mathrm{s}$; the crosses, rectangles and reversed triangles represent the wave shape drag under the same windspeeds. Near the wind wave interface, turbulence plays a dominant role, and the shape drag due to significant wave is small. The wave surface roughness $z_{\mathrm{s}}$ represents short wave contribution to the momentum exchange, so the turbulence friction drag can be regarded as the addition of interface friction and impact of short waves. It is difficult to decompose surface friction, waves impact and turbulence from interface momentum flux. In fact, the three of them interact, and it is impossible to decompose them thoroughly from the physical aspect. According to fig. 4 the shape drag has a small value. Significant wave represents the influence of long waves on momentum exchange, so the long waves change the large-scale structure of turbulence but affect momentum exchange at the air-sea interface weakly. In the figure the friction drag has larger values, which means that the change of small scale turbulence structure due to short waves has a larger impact. The value of the shape drag even can be negative when wind waves are developed because the significant wave speed is a little bit larger than the $10 \mathrm{~m}$ high windspeed.

The variation of the drag coefficient $C_{\mathrm{D}}$ with wave development is shown in fig. 5, in which curves $1-3$, diamonds and triangles represent windspeeds of 5,10 and $15 \mathrm{~m} / \mathrm{s}$ respectively. It can be concluded from fig. 5 that the drag coefficient $C_{\mathrm{D}}$ decreases with the wind wave development. In the initial period, most of the wind waves are high frequency components which have large

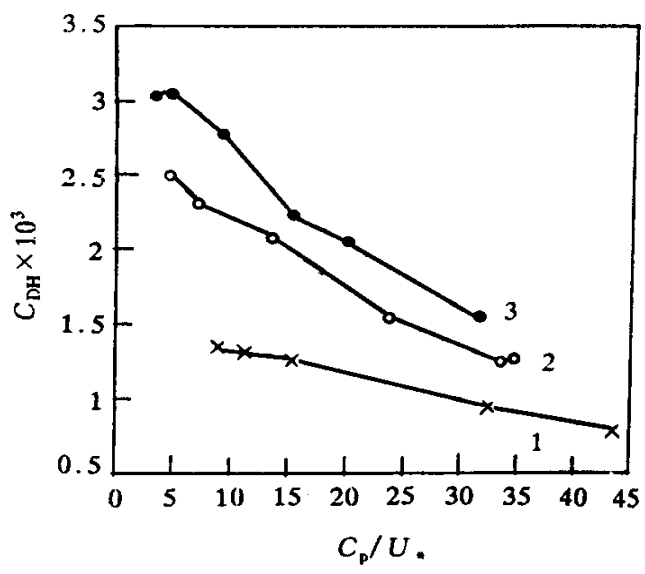

Fig. 5. Variation of the drag coefficient $C_{\mathrm{D}}$ with wave age $C_{\mathrm{p}} / U$ * 
slope and low wave speed, so the drag coefficient has a larger value. With the development of wind waves, the wave height, wave length and wave speed increase but the wave slope decreases, which implies the reduction of the drag coefficient. From the figure it can be concluded that $C_{\mathrm{D}}$ decreases linearly as wave age increases. The results are compared with the measurement by Donelan ${ }^{[1]}$ in fig. 6 . The diamonds, dots, triangles, crosses and multipliers, represent the ranges of $U / C_{\mathrm{p}}$. They are $0.8-1.5,1.5-2.0,2.0-3.0,3.0-4.0,4.0-6.0$, respectively. The two lines with circles and triangles mean the variation of $C_{\mathrm{D}}$ when $U * / C_{\mathrm{p}}$ is 0.2 and 0.04 , respectively. Donelan's data were obtained in Lake Ontario, which only have finite fetches. However, during the initial period of wave developing, the scales of waves are not so large that the influence of the lake bottom and border can be neglected. Then the interaction of wind waves on lake is almost the same as the deep sea. Although the distribution of observational data is not very regular in the figure, it still can be concluded that the result of numerical simulation well fits in with the observation.
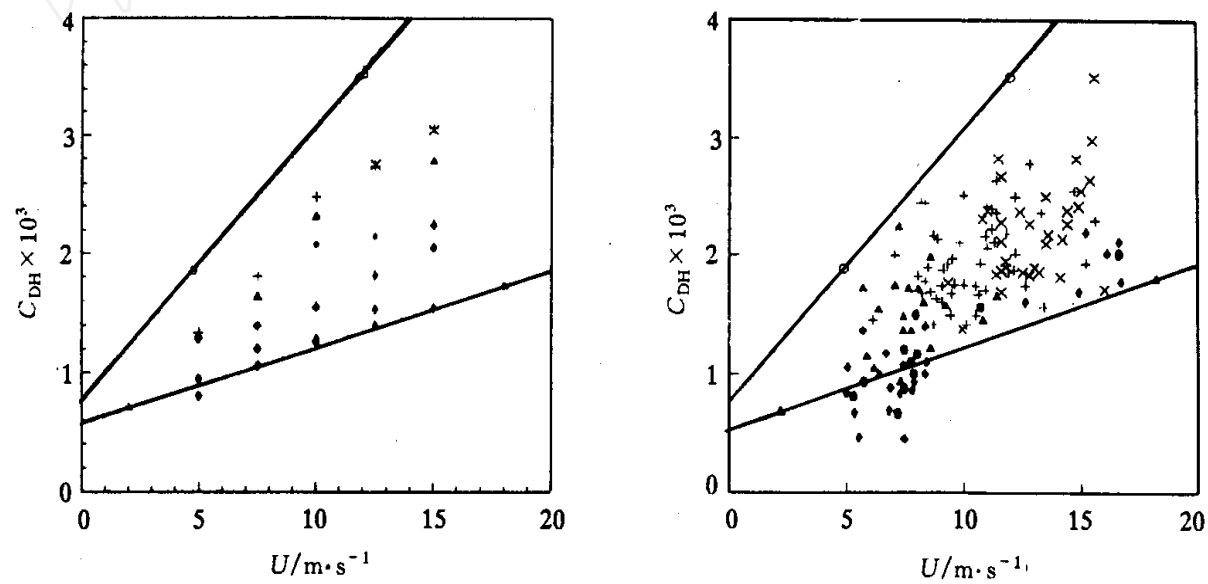

Fig. 6. Comparison of calculating result with Donelan's observing result.

\section{Conclusion}

In order to study the influences of wave development on air-sea interaction, a new model of wind waves is used in numerical simulation. The significant wave and surface roughness represent the influence of waves on air flow , $k-\epsilon$ model is adopted for turbulence simulation. Then air turbulence above waves with wave development is simulated. The variation of the drag coefficient with wave age agrees well with the observations. The JONSWAP spectrum is used to determine the parameters of the interface model including significant wave and surface roughness. However, the adoption of different wave spectrum would change the values of the interface model. A formula of wave surface roughness is obtained based on dimensional analysis and result comparison. The accuracy should be testified by further theoretical study and measurement. When windspeed exceeds $15 \mathrm{~m} / \mathrm{s}$, wave breaking which partly alters the process of momentum transfer will occur frequently. Therefore, a further improvement of this model is absolutely needed. 


\section{References}

1 Donelan, M. A. , The dependence of the aerodynamic the drag coefficient on wave parameters, First International Conference on Meteorology and Air-sea Interaction of the Coastal Zone, Boston: Am. Meteorol. Soc. , 1982, $381-387$.

2 Charnock, H., Wind stress on a water surface, Q. J. R. Meteorol. Soc., 1955, 81: 639.

3 Maat, N. , Kraan, C. , Oost, W. A. , The roughness of wind waves, Boundary-layer Met. , 1991, $54: 89$.

4 Kitaigorodskii, S. A., The Physics of Air-Sea Interaction (trans. Baruch, A.), Jerusalem: Israel Program of Scientific Translations, 1973.

5 Geernaert, G. L., On the importance of the drag coefficient in air-sea interactions, Dynamics of Atmospheres and Oceans, $1987,11: 19$.

6 Geernaert, G. L. , Variation of the drag coefficient and its dependence on sea state, Ph. D. Dissertation, Seattle : University of Washington, 1983, 203.

7 Geernaert, G. L., Drag coefficient modeling for the near coastal zone, Dynamics of Atmospheres and Oceans, 1988, 11: 307.

8 Makin, V. K. , Kudryavtsev, V. N. , Mastenbroek, C. , Drag of the sea surface, Boundary-layer Met. , $1995,73: 159$.

9 Chalikov, D. V. , The numerical simulation of windwave interaction, J. Fluid Mech. , 1978, 87: 561.

10 Chalikov, D. V. , Numerical simulation of the boundary layer above waves, Boundary-layer Met. , 1986, $34: 63$.

11 Le Ngocly, An application of the $E-\epsilon$ turbulence model for studying coupled air-sea boundary layer structure, Boundary-layer Met. , 1991, $54: 327$.

12 Maat, N. , Makin, V. K. , Numerical simulation of air flow over breaking waves, Boundary-layer Met. , $1992,60: 77$.

13 Zhang Zifan, Li Jiachun, Numerical study on drag coefficient of air over well-developed wind waves, Acta Mechanica Sinica, $1997,29: 385$ 